\title{
Validity and Reliability of the Brief Scale of Alcohol Dependence (BEDA) on Teenagers
}

\author{
Jesús Tánori Quintana ${ }^{1}$, Gildardo Bautista Hernandez ${ }^{2}$, Jose Angel Vera Noriega ${ }^{2}$, Angel Alberto Valdes \\ Cuervo $^{1}$, Christian Oswaldo Acosta Quiroz ${ }^{3}$ \& Daniela Hernández Ramos ${ }^{4}$ \\ ${ }^{1}$ Department of Education, Instituto Tecnológico de Sonora, Ciudad Obregón, Sonora, México \\ ${ }^{2}$ Regional Development Coordination, Centro de Investigación en Alimentación y Desarrollo, A.C., Hermosillo, \\ Sonora, México \\ ${ }^{3}$ Department of Psychology, Instituto Tecnológico de Sonora, Ciudad Obregón, Sonora, México \\ ${ }^{4}$ Mental Hygiene Hospital, Carlos Nava, Secretaria de Salud, Hermosillo, Sonora, México \\ Correspondence: Jesús Tánori Quintana, Department of Education, Instituto Tecnológico de Sonora, Ciudad \\ Obregón, Sonora, México. E-mail: jesus.tanori@itson.edu.mx
}

Received: October 14, 2016

Accepted: November 20, $2016 \quad$ Online Published: December 5, 2016

doi:10.5539/jedp.v7n1p36

URL: http://dx.doi.org/10.5539/jedp.v7n1p36

\begin{abstract}
The Brief Scale of Alcohol Dependence (BEDA) has shown validity and reliability on previous studies. Despite the frequent use in treatment programs for addictions, there are gaps on its psychometric properties and diagnostic efficiency on the teenage population. This study conducted analysis of construct validity of the Brief Scale of Alcohol Dependence and its relation to attitudes towards an accident prevention program for young people in Sonora. 838 high school students (Cecytes) from Hermosillo participated, including students from second, fourth and sixth semesters. 54\% are male, while the rest (46\%) are female, with an average age of 16 and a SD of 1 , with a minimum age of 15 and a maximum of 19 . Results show that the scale meets the statistical criteria that proves its validity and supports its use on the teenage population. Results support the hypothesis of the scale having evidence of validity and reliability, which justifies its use in research and in the creation of indicators of alcohol consumption in young people.
\end{abstract}

Keywords: validity and reliability, brief scale, alcohol dependence

\section{Introduction}

The importance of monitoring substance use among students offers the possibility of assessing risk behaviors of today's youth (European Monitoring Centre for Drugs and Drug Addiction, 2012). This practice can be carried out in Mexico by means of the National Survey of Addictions (ENA), which allows analyzing data on different age ranges. It has been recorded in the adolescent population that alcohol consumption has significantly increased according to different parameters: consumed alcohol once increased from $35.6 \%$ to $42.9 \%$; consumed in the last year increased from $25.7 \%$ to $30.0 \%$, and consumed in the last month from $7.1 \%$ to $14.5 \%$. This trend was observed in men and women, especially in consumed in the last month, going from $11.5 \%$ to $17.4 \%$ for men and from $2.7 \%$ to $11.6 \%$ for women Villatoro-Velázquez et al. (2012).

A comparison of the 2008 and the 2011 ENAs reveals that the user rate registered a significant increase among young people from $2.1 \%$ to $4.1 \%$, while high consumption (daily) stayed the same and the regular drinker decreased from $2.2 \%$ to $1 \%$. Gender-wise, daily consumption held low percentages for both genders, regular use decreased and dependence doubled in males (from 3.5\% to 6.2\%) and tripled in females from .2 to $.6 \%$.

The data obtained from the ENA and the Encode show the magnitude of the problem of alcoholism, which threatens an important part of Mexico's young population; as physical, social, and psychological consequences associated to the use, abuse, and dependence on alcohol is a public health problem (Guzmán, Pedrao, Rodríguez, López, \& Esparza, 2007; Mora \& Natera, 2001).

Studies related to addictions in young people are based on the fact that it is precisely at this stage of life in which consumption of alcohol, tobacco, and other drugs most often starts. The average age of onset of alcohol consumption is 16 , however, in recent years consumption occurs at a younger age (Martínez, Salazar, Pedroza, 
Ruiz, \& Ayala, 2008). This implies a greater likelihood of developing dependence, and so here lies the need to understand the factors associated to the onset and form of alcohol use in this age group (Cadaveira, 2009; Maradiegue, 2010).

Likewise, excessive use of alcohol is considered as one of the five main risk factors for illness, impairment, and death worldwide. For instance, in 2012, 5.9\% of all deaths worldwide (3.3 million) were due to the consequences of using this substance (Villatoro-Velázquez et al., 2014).

One of the main causes of death are those that link alcoholism and car accidents. This bond has brought about the implementation of the Designated Driver Program (PCD) since August $7^{\text {th }} 1992$, as a preventive measure against alcohol related car accidents and to promote awareness in the community about the importance of driving sober, thus encouraging a culture of responsible consumption. However, encouraging a culture of responsible consumption would be on a par with a change in the beliefs and reasoning system that allow the young person to realize the risk involved in alcohol consumption, in order for them to reduce drinking and avoid building alcohol dependence and a disorder.

This raises a methodological need related to the presence of an instrument that facilitates the detention of teenage students at risk with alcohol. This effort has been made in some States in the country with different scales. For example, the scale of Alcohol Use Disorders Identification Test (Audit) was validated for high school students in Mexico City (Gómez, Gómez, Pérez, \& Morales, 2009).

Another study carried out in Mexico (Pérez-López, Villalobos-Gallegos, Viveros, Graue-Moreno, \& Marín-Navarrete, 2015). Validated three different versions of the BEDA (15, 14, and 12 items), by means of a confirmatory factor analysis, but with people who were receiving care in residential treatment centers for addictions, concluding that the three versions presented an internal consistency $>.90$, adequate goodness of fit, and significant correlations with the Mini International Neuropsychiatric Interview, v. 5.0, of diagnostic criteria of Alcohol Dependence (DA).

\subsection{Objetive}

This study carried out validity and reliability analysis of the Brief Scale of Alcohol Dependence and its relation with attitudes toward an accident prevention program for young people.

\section{Method}

\subsection{Participants}

838 high school students (Cecytes) from Hermosillo participated, including students from second, fourth and sixth semesters. $54 \%$ are male, while the rest (46\%) are female, with an average age of 16 and a SD of 1 , with a minimum age of 15 and a maximum of 19.

\subsection{Instruments}

\subsubsection{Brief Scale of Alcohol Dependence (BEDA)}

The Echeverría, Ruiz, Tiburcio y Ayala (2007) version was used. It consists of 15 items that are answered in a continuum of Never-Almost always. The scale's aim is to measure dependence in the adult population and it was adapted to be applied to youths.

\subsubsection{Attitude and Knowledge of the Designated Driver Program}

Developed especially for this study, by means of the elements and criteria in which the designated driver program is based on, consists of 10 items made with Likert response options that retrieve the agreement-disagreement attitudes, and it is based in the knowledge one may have about the program.

\section{Procedure}

The data was gathered by the implementation of a group questionnaire, requesting prior authorization of the relevant directors and the informed consent of the participants.

Data analysis consisted of an exploratory factor analysis with half of the participants, employing the maximum likelihood extraction method and Oblimin rotation in SPSS 20, and then a confirmatory factor analysis with the structural equations method using AMOS. 


\section{Results}

\subsection{Factor Analysis}

An exploratory factor analysis was performed to the BEDA, achieving one-dimensionality with values of .922 in the Kaiser-Meyer-Olkin measure of sample adequacy; and a significant Bartlett test of sphericity $\left(X^{2}=4491.29\right.$, $p=.000$ ), which held data adequacy for this type of analysis (Hair, Black, Babin, \& Anderson, 2006).

The criterion for including the dimension in the factorial solution was that at least $10 \%$ of the variance was explained, and as for items, that they present factor loadings equal or greater than .30 (Martínez, Hernández, \& Hernández, 2009). The final solution consisted of 11 items, excluding four, which characterize the young respondents, since those that were not significant are basically related to the possibility to continue drinking and to the freedom to do so without accounting to anyone, for instance: do you drink your desired amount regardless of what you have to do the following day? Moreover, the values of explained variance and internal consistency rate alike turned out to be adequate, which indicates strong evidence of construct validity.

Likewise, an exploratory factor analysis was carried out for the attitudes towards the designated driver program resulting two factors as theoretically designed, with values of $K M O=.859$; and a significant Bartlett test of sphericity $\left(X^{2}=1181.98, p=.000\right)$, which showed data adequacy for this type of analysis.

The final solution consisted of seven items: four items distributed in factor one, which has been denominated congruous attitude towards the program and explains $47.39 \%$ of the variance, and three items in factor two, which has been denominated incongruous attitude towards the program and explains $9.83 \%$ of the variance, as well as the internal consistency rate (Cronbach's Alpha $=.89$ ), which shows strong evidence of construct validity.

Table 1. Brief Scale of Alcohol Dependence (BEDA)

\begin{tabular}{llc}
\hline \multicolumn{1}{c}{ Items/Brief Scale of Alcohol Dependence (BEDA). } & $\mathrm{h}^{2}$ & Factor Loading \\
\hline R1. Do you have difficulty to stop thinking about drinking? & .53 & .73 \\
R2. Is drinking in excess more important than your next meal? & .53 & .73 \\
R3. Do you organize your day according to where and when you may drink? & .42 & .65 \\
R4. Do you drink in the morning, afternoon, and night? & .53 & .48 \\
R5. Do you drink for the effect of alcohol regardless of what beverage you are drinking? & .54 & .69 \\
R10. The next morning after drinking too much, do you feel the need to drink in order to function? & .58 & .73 \\
R11. The next morning after drinking too much, do you wake up with shaky hands? & .58 & .76 \\
R12. After drinking a large amount, do you wake up and vomit? & .50 \\
R13. The next morning after drinking too much, do you avoid people? & .59 \\
R14. After drinking too much, do you see things that scare you even when you realize its your imagination? & .76 \\
R15. Do you go out for a drink and the next day forget what happened the previous night? & .44 & .70 \\
& \% of the variance & .67
\end{tabular}

Note. $\mathrm{h}^{2}=$ communality.

Table 2. Attitude and knowledge of the designated driver program

\begin{tabular}{|c|c|c|c|}
\hline & \multicolumn{3}{|c|}{ Factor Loading } \\
\hline & $h^{2}$ & $\mathrm{~F} 1$ & $\mathrm{~F} 2$ \\
\hline $\begin{array}{l}\text { R5. The main goal of the designated driver is: to drive all of his friends back home safe and } \\
\text { sound. }\end{array}$ & .73 & .85 & -.52 \\
\hline $\begin{array}{l}\text { R3. The Designated Driver Program raises awareness about the importance of not mixing } \\
\text { alcohol and driving, promoting a responsible consumption culture in the community. }\end{array}$ & .63 & .79 & -.44 \\
\hline R7. The largest commitment of a designated driver is to not drink alcoholic beverages. & .63 & .79 & -.52 \\
\hline
\end{tabular}


R1. Designated Driver is a program at a national level that contributes to the reduction of car accidents involving alcohol abuse.

.55

R8. Another major commitment of the designated driver is to notify other drivers about police checkpoints.

R10. Being a designated driver allows you to be a police officer, in the near future, without taking any tests.

R4. The extended hours of alcohol sales favors the caring of people in the community and makes it less likely for people to mix alcohol and driving.

$\begin{array}{lll}.60 & -.48 & .77\end{array}$

$\begin{array}{lll}.55 & -.39 & .73\end{array}$

.28

$-.42$

.51

Note. $\mathrm{h}^{2}=$ communality.

\subsection{Confirmatory Factor Analysis}

From the results of the exploratory factor analysis, a model for measuring the Alcohol Dependence construct was proposed, in which the presence of a one-dimensional factor was established and related to a congruous or incongruous attitude toward the designated driver program as evidence of divergent validity.

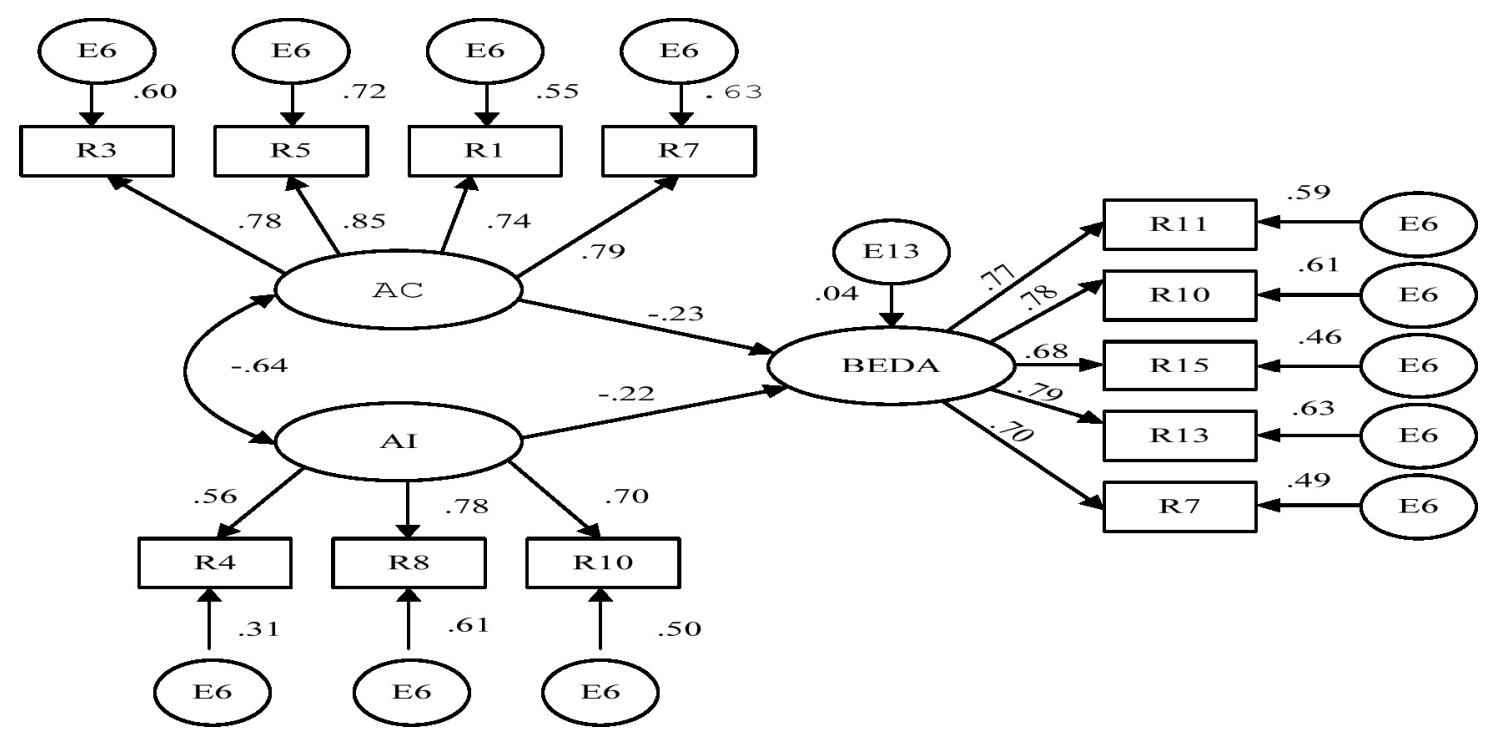

Figure 1. Theoretical model for measuring alcohol dependence and knowledge and attitudes toward an intervention intention of alcohol consumption

The estimation procedure of the model used was that of maximum likelihood based on the $X^{2}$ test. Absolute fit, incremental, and parsimony rates were taken into account to evaluate the model's goodness of fit to the data (Cea, 2004; Hair, Black, Babin, \& Anderson, 2006). The absolute fit value is given by the statistical $X^{2}$, that in this case the hypothesis $\left[X^{2}(51)=96.674, \mathrm{p}<.001\right]$ is rejected, however the rate $\operatorname{CMIN}\left(X^{2} / g l\right)=1.89$, the value of $R M S E A=.04$, the incremental fit rate $\mathrm{NFI}=.955$ and the $\mathrm{CFI}=.978$ and the parsimony rate, $\mathrm{PNFI}=.738$, suggest that the model gained a satisfactory general fit (see Figure 1).

\section{Discussion}

The need to value health or conditions of well being in the population, regardless of age or social distinction, is a priority for governments, with population surveys being one of the most efficient strategies. Surveys, on the other hand, must have some very important features, such as: validity, reliability, and prompt resolution (Villatoro-Velázquez et al., 2015).

The results support the hypothesis that the scale shows evidence of validity and reliability, which justify its use in research and creating indicators of alcohol consumption in young people. The value of the scale is reinforced by the solidity of the studies that report the existence of indicators in the adult population (Gómez, Gómez, Pérez, 
\& Morales, 2009). On the other hand, small correlations between the dimensions of attitude, which manifest knowledge about the designated driver program, assert that it is of little importance how much is known about the program and that its relation with consumption is scant and in a directly proportional sense. This suggests that the more is known about the program, the greater the alcohol consumption is. While this is beneficial, because car accidents are reduced, addictions can be brought up among young people.

Although the findings suggest that the scale is a valuable tool for measuring the construct, it must be stated that it has limitations, being that only the usual items were worked with. In this sense it is recommended to write new items that are related to the socialization of young people nowadays.

\section{Acknowledgments}

This study is part of the project "Design and Evaluation of Preventive and Corrective Programs Related to the use of Alcohol in Teenagers and Young People" (Diseño y Evaluación de Programas Preventivos y Correctivos Relacionados con el uso del Alcohol en Adolescentes y Jóvenes), financed by the Hermosillo municipal government, March-July 2012. The authors wish to thank the Hermosillo Municipal Government 2009-2012.

\section{References}

Cadaveira, F. (2009). Alcohol y cerebro adolescente [Alcohol and teenage brain]. Adicciones, 21(1), 9-14. https://dx.doi.org/10.20882/adicciones.246

Cea, M. (2004). Multivariate analysis. Theory and practice in social research. España: Síntesis.

European Monitoring Centre for Drugs and Drug Addiction. (2012). The state of the drugs problem in Europe. Luxembourg: Publications Office of the European Union.

Gómez, E., Gómez, H., Morales, B., \& Pérez, M. (2009). Using AUDIT and DAST-10 for identifying abuse of psychoactive substances and alcohol in adolescents. Revista Colombiana de Psicología, 18(1), 189.

Guzmán, F., Pedrao, L., Rodríguez, L., López, K., \& Esparza, E. (2007). Alcohol Consumption Disorder (AUDIT) for Marginal Adolescents and Youth from Juvenile Gangs of Mexico. Revista de Enfermagen, 11(4), 611-618.

Hair, J., Black, W., Babin, B., \& Anderson, R. (2006). Multivariate Data Analysis (7th ed.). Nueva Jersey: Pearson.

Maradiegue, A. (2010). Central American mothers report family history of depression and alcohol abuse a predictor of teenage health risk behaviors. Journal of the American Association of Nurse Practitioners, 22(10), 540-547. https://dx.doi.org/10.1111/j.1745-7599.2010.00548.x

Martínez, K., Salazar, M., Pedroza, F., Ruiz, G., \& Ayala, H. (2008). Preliminary results of the brief intervention program for teenagers who start using alcohol and other drugs. Salud Mental, 31(2), 119-127. Retrieved from http://www.revistasaludmental.mx/index.php/salud_mental/article/view/1218/1216

Martínez, M., Hernández, M., \& Hernández, M. (2009). Psychometry. Madrid: Alianza Editorial.

Mora, J., Natera, G., \& Juárez, F. (2001). Expectations, alcohol consumption and related problems in university students in Mexico City. Salud Pública, 43(2), 89-96. Retrieved from http://bvs.insp.mx/rsp/_files/File/2001/VOL\%2043\%202/v43_2_expectativas.pdf

Pérez-López, A., Villalobos-Gallegos, L., Viveros-Rodríguez, M., Grague-Moreno, J., \& Marín-Navarrete, R. (2015). Three versions of the Short Alcohol Dependence Data Questionnaire (SADD) in Mexican population: A comparative analysis of psychometric properties and diagnostic accuracy. Salud Mental, 38(4), 281-286. https://dx.doi.org/10.17711/SM.0185-3325.2015.038

Villatoro-Velázquez, J., Fregoso, D., Bustos, O., Mujica, A., Martin del Campo, R., Alvarado, N., \& Medina-Mora, M. (2015). National Survey of Drug Use in Students. México. 2014. Alcohol Report. Instituto Nacional de Psiquiatría Ramón de la Fuente Muñiz: Comisión Nacional Contra las Adicciones: Secretaría de Salud.

Villatoro-Velázquez, J., Medina-Mora, M., Fleiz-Bautista, C., Téllez-Rojo, M., Mendoza-Alvarado, L., Romero-Martínez, M., \& Gutiérrez-Reyes, J. (2012). National Survey of Addictions 2011: Alcohol Report. México: Instituto Nacional de Psiquiatría Ramón de la Fuente Muñiz: Comisión Nacional Contra las Adicciones: Secretaría de Salud. 


\section{Copyrights}

Copyright for this article is retained by the author(s), with first publication rights granted to the journal.

This is an open-access article distributed under the terms and conditions of the Creative Commons Attribution license (http://creativecommons.org/licenses/by/4.0/). 